\title{
Consumo de fibra dietética, sodio, potasio y calcio y su relación con la presión arterial en hombres adultos normotensos
}

Martha N ydia Ballesteros-Vásquez, Q.B., M. en C., ${ }^{(1)}$ Rosa María Cabrera-Pacheco, Q.B., M. en C., ${ }^{(1)}$ María del Socorro Saucedo-Tamayo, Lic. en Enf., (1) María Isabel Grijalva-Haro, Q .B., M. en C.(1)

Ballesteros-Vásquez MN, Cabrera-Pacheco RM, Saucedo-Tamayo MS, Grijalva-Haro MI. Consumo de fibra dietética, sodio, potasio y calcio y su relación con la presión arterial en hombres adultos normotensos. Salud Publica Mex 1998;40:241-247.

\section{Resumen}

Objetivo. Evaluar el efecto del consumo de fibra dietética, energía, sodio, potasio y calcio sobre la presión arterial de un grupo de adultos normotensos, tomando en cuenta indicadores tales como la edad, el sexo, la actividad física y la obesidad. Material y métodos Se evaluaron 38 sujetos del sexo masculino de 30 a 45 años de edad, normotensos, aparentemente sanos y residentes de la ciudad de Hermosillo, Sonora, México. Se midió la presión arterial y se realizó una evaluación dietética, antropométrica y de actividad física. Resultados La dieta resultó ser alta en fibra y en grasa. El sodio estaba $56 \%$ por arriba de la recomendación en $87 \%$ de los casos y fue la variable que más efecto mostró sobre la presión diastólica. De los sujetos estudiados, $36.9 \%$ tenían sobrepeso y obesidad, y se encontró una asociación significativa entre el índice de masa corporal y la presión diastólica y sistólica. Conclusiones Existe una asociación significativa entre la hipertensión arterial y el alto consumo de sodio, el sobrepeso y la obesidad en sujetos normotensos.

Palabras clave: presión sanguínea; fibra en la dieta; sodio en la dieta; potasio en la dieta; calcio en la dieta; México
Ballesteros-Vásquez MN, Cabrera-Pacheco RM, Saucedo-Tamayo MS, Grijalva-Haro MI.

Dietary fiber, energy, sodium, potassium and calcium intake and its relationship to blood pressure in normotensive male adults. Salud Publica Mex 1998;40:241-247.

\begin{abstract}
A bstract
Objective. To evaluate the effect of dietary fiber, energy, sodium, calcium and potassium intake on the blood pressure of a group of normotensive adults. Material and methods Subjects were 38 healthy normotensive adult males aged 30-45 years, resident of Hermosillo, Sonora, Mexico. Blood pressure, diet, anthropometric data and physical activity were recorded. Results. Their diet was high in fiber and fat.Sodium intake was $56 \%$ above the recommended values in $87 \%$ of the subjects, and the variable with the highest correlation to diastolic blood pressure. $0 \mathrm{f}$ the subjects, $36.9 \%$ were overweight and obese, and a significant statistic association was found between BMI and diastolic and systolic blood pressure. Conclusions. A significant relationship was found between hypertension and high consumption of sodium, overweight and obesity in healthy adult normotensive males.
\end{abstract}

Key words: blood pressure; fiber, dietary; sodium, dietary; potassium, dietary; calcium, dietary; Mexico

(1) Centro de Investigación en Alimentación y Desarrollo,A.C., Hermosillo, Sonora, México.

Fecha de recibido: 30 de junio de 1997 - Fecha de aprobado: 3 de marzo de 1998 
$L$ a hipertensión arterial es uno de los principales factores de riesgo para desarrollar enfermedad cardiovascular; diversos estudios han notificado algunos factores que son conducentes a la aparición de la hipertensión arterial como los de orden genético, la edad, el estrés, el índice de Quetelet y los dietéticos como algunos cationes, entre los que destacan el sodio, el potasio y el calcio. ${ }^{1,2}$

De todos esos factores se considera que el más riesgoso es la ingestión excesiva de sodio, ${ }^{3}$ cuya relación con la presión arterial es complicada y controversial; por un lado, algunos estudios epidemiológicos han demostrado que existe una relación directa entre estas dos variables y, por el otro, algunas investigaciones de intervención no han podido obtener un efecto convincente respecto a la noción de que al reducir la ingestión de sal se altera la presión arterial. ${ }^{4,5}$

Al parecer, aproximadamente una cuarta parte de los sujetos normotensos y alrededor de la mitad de los pacientes hipertensos son caracterizados como sensibles al sodio, esto es que el exceso de sodio en la dieta aumenta su presión arterial. ${ }^{6}$ En este contexto el informe INTERSALT, ${ }^{7,8}$ que incluyó estudios llevados a cabo en 52 países, notificó que una ingestión de sal de $2.4 \mathrm{~g} / \mathrm{d}(100 \mathrm{mmol} / \mathrm{d})$ aumenta la presión arterial. La Organización Mundial de la Salud (OMS) ${ }^{9}$ y la National Academy of Science (NAS) ${ }^{10}$ en los Estados Unidos de América (EUA) recomiendan que el consumo de sodio se reduzca a $2.4 \mathrm{~g} / \mathrm{d}$ máximo.

Existen informes donde se establece que el potasio puede estar vinculado con la presión arterial; por otra parte, algunos estudios epidemiológicos han mostrado que la hipertensión es prevalente en poblaciones cuya dieta es alta en sodio y baja en potasio. ${ }^{11,12}$ Una ingestión elevada de potasio no altera la presión arterial en sujetos normotensos, pero puede bajarla en sujetos hipertensos. ${ }^{13-15}$

De acuerdo con algunos autores ${ }^{16,17}$ el calcio desempeña un papel más activo en la hipertensión que el sodio y el potasio; la hipótesis es que la ingesta insuficiente de calcio en la dieta solo o combinado con otros factores, predispone a la hipertensión arterial.

Otros componentes dietéticos con un posible efecto sobre la presión arterial son el alcohol, ${ }^{18}$ el coleste$\mathrm{rol}^{19}$ y la fibra; de esta última, la observación nace a partir de que se ha mostrado que los sujetos vegetarianos, cuya dieta incluye grandes cantidades de fibra, tienen una presión arterial menor que los sujetos omnívoros. ${ }^{20}$ De igual manera, en algunos estudios se ha demostrado que el incremento en la ingestión de fibra dietética provoca una disminución en la presión arterial; ${ }^{21}$ no obstante, a pesar de esa evidencia el tema aún es controversial ya que en otros estudios no se ha encontrado ninguna asociación. ${ }^{22}$

El impacto de los cambios dietéticos sobre la presión arterial se ha estudiado ampliamente en sujetos hipertensos y muy poco en normotensos; se ha informado que la reducción en el consumo de sodio en sujetos normotensos de 3.6 a $1.5 \mathrm{~g} / \mathrm{d}$ provoca una disminución de hasta $2 \mathrm{mmHg}$ en la presión sistólica y diastólica. Asimismo, la suplementación con $1.5 \mathrm{~g} / \mathrm{d}$ de calcio en sujetos normales disminuye la presión arterial. ${ }^{23}$

Las estadísticas nacionales de salud indican que la hipertensión arterial se considera un problema de salud pública; la prevalencia más elevada se presenta en la región norte del país, ${ }^{24}$ particularmente en el estado de Sonora, México. La hipertensión arterial esencial es en la entidad una de las veinte principales causas de morbilidad general con una tasa de 171.9/100 000 habitantes. ${ }^{25}$

De acuerdo con lo anterior, el objetivo de esta investigación es el de evaluar el efecto del consumo de fibra dietética, sodio $(\mathrm{Na})$, potasio $(\mathrm{K})$, calcio $(\mathrm{Ca})$, grasa y alcohol sobre la presión arterial de un grupo de adultos normotensos, tomando en cuenta indicadores como la edad, el sexo, la actividad física y la obesidad.

\section{Material y métodos}

\section{Población de estudio}

Para seleccionar a los sujetos del estudio se llevó a cabo un muestreo intencional no probabilístico, en el que participaron 126 hombres de nivel socioeconómico medio y bajo, ${ }^{26}$ residentes de la ciudad de Hermosillo, Sonora, México. Del total de sujetos evaluados sólo 38 cumplieron con las condiciones del protocolo, esto es, ser normotensos, del sexo masculino, en edades de 30 a 45 años (por ser éste el grupo con mayor riesgo de sufrir hipertensión y enfermedad cardiovascular) ${ }^{27,28} \mathrm{y}$ que aparentemente no padecieran diabetes mellitus ni enfermedad cardiovascular; los datos se obtuvieron aplicando un breve cuestionario clínico diseñado para este caso.

$\mathrm{Al}$ momento de llevar a cabo el estudio ninguno de los sujetos consumía medicamentos ni complementos vitamínicos, de minerales o fibra. No se les restringió la actividad física antes ni durante el periodo del estudio, así como tampoco la alimentación. El Comité de Etica del Centro de Investigación en Alimentación y Desarrollo, A.C., aprobó el protocolo, y los participan- 
tes voluntarios firmaron una hoja de consentimiento de acuerdo con las guías institucionales. El estudio se realizó de marzo de 1995 a octubre de 1996.

\section{Presión arterial}

Se midió la presión arterial de los sujetos en dos ocasiones, siguiendo las recomendaciones estándar de la $\mathrm{OMS}^{29}$ y utilizando su clasificación de presión arterial sistólica (PAS $<140 \mathrm{mmHg}$ ) y de presión arterial diastólica $(\mathrm{PAD}<90 \mathrm{mmHg})$. En la medición se utilizó un baumanómetro de columna de mercurio (Desk Model Adult Calibrated 0320, distribuido por Graham-Field Inc., Nueva York, EUA, y fabricado en Japón). Durante el procedimiento el sujeto permaneció sentado cómodamente durante 10 minutos antes de que se tomara la lectura; posteriormente con el antebrazo izquierdo ligeramente separado del cuerpo y descansando sobre una mesa, semiextendido y con la palma de la mano hacia arriba, se tomaron dos lecturas, para promediar el valor reportado. Se encontró que todos los sujetos eran normotensos.

\section{Evaluación dietética}

Para evaluar el consumo de nutrimentos se utilizó el método de registro de peso de los alimentos por siete días. ${ }^{30}$ Los participantes recibieron entrenamiento tanto para llenar el formato de registro, como para pesar los alimentos; para esta última operación se les proporcionó una balanza digital (capacidad de $1 \mathrm{~kg}$ Ohaus Modelo Lume O Meter; Nueva Jersey, EUA). Se registró cada alimento o bebida consumida en casa o fuera de ella de la misma manera. Para la cuantificación de nutrimentos se utilizó un programa de computación, ${ }^{31}$ que incluye datos de análisis de alimentos regionales así como tablas de composición de alimentos extranjeras y nacionales. Para este estudio se utilizó la base de datos de alimentos regionales y americanos, por seguir la misma metodología analítica para los nutrimentos estudiados. . $2,33^{2}$

\section{Evaluación antropométrica}

El peso y la estatura se midieron según la técnica de Jellife \& Jellife, ${ }^{34}$ utilizando una balanza de plataforma con capacidad de $150 \pm 0.05 \mathrm{~kg}$ (Accu Weigth, Mod Health O Meter, distribuida por Metro Equipment Corp., EUA, y fabricada en Alemania). La estatura se midió con una cinta métrica de fibra de vidrio de $150 \mathrm{~cm}$ de longitud y una escuadra de madera; esta metodología se estandarizó o $^{34}$ con un estadiómetro
Holtain (Holtain Ltd, Inglaterra). El índice de masa corporal $\left(\mathrm{kg} / \mathrm{m}^{2}\right)$ se calculó en todos los sujetos como indicador de obesidad y siguiendo la clasificación de NHANES II. ${ }^{35}$

\section{Actividad física}

El registro de actividad física de siete días se utilizó para medir todas las actividades realizadas durante el día. ${ }^{36} \mathrm{~A}$ las actividades registradas se les asignó un valor en términos de múltiplos del metabolismo basal $(\mathrm{mMB})$; la estimación del metabolismo basal se llevó a cabo mediante las ecuaciones de FAO/OMS/UNU, ${ }^{37}$ de acuerdo con el peso corporal, el sexo y la edad. La actividad también se clasificó de acuerdo con la $\mathrm{FAO}^{37}$ como ligera cuando era de $1.55 \mathrm{mMB}$; moderada, de $1.78 \mathrm{mMB}$, y de actividad intensa, $2.10 \mathrm{mMB}$. Los sujetos registraron las actividades por lapsos de 15 minutos, y se sumó el tiempo total gastado en cada actividad durante siete días.

\section{A nálisis estadísticos}

Se calculó la media y la desviación estándar del consumo de nutrimentos. Asimismo se realizó un análisis de regresión lineal simple, utilizando la presión diastólica y sistólica como variables dependientes, y la edad, el consumo de fibra dietética, energía, $\mathrm{Na}, \mathrm{K}, \mathrm{Ca}$, relación $\mathrm{Na}-\mathrm{K}$, actividad física, peso e índice de masa corporal (IMC), así como consumo de grasa y colesterol, como variables independientes. De todas estas variables, las que presentaron una relación significativa se pusieron en un modelo de regresión múltiple por pasos con el fin de obtener la variable que presentara mayor efecto sobre ambas presiones. Para las pruebas se utilizó el paquete estadístico Statgraphics, versión 4.0, 1989.

\section{Resultados}

De acuerdo con la edad de los sujetos estudiados éstos pueden considerarse como adultos jóvenes; siguiendo los criterios establecidos por la OMS en cuanto a hipertensión arterial, se clasificaron en promedio como normotensos (cuadro I). Sin embargo, es importante mencionar que de los 38 individuos evaluados siete presentaron presión sistólica entre 130-140, y 14, presión diastólica entre 85-95, rangos que pueden considerarse como presión normal alta.

Respecto a la evaluación antropométrica los resultados indicaron que el valor promedio del IMC obtenido fue de 27.3. En la figura 1 se muestra la gráfica de 
Cuadro I

Características de los sujetos del estudio sobre COMPONENTES DIETÉTICOS Y PRESIÓN ARTERIAL. Hermosillo, Sonora, México, 1995-1996

Media $\pm D E(n=38)$

\begin{tabular}{lc} 
Edad (años) & $37 \pm 4$ \\
\hline Presión arterial & \\
\hline Sistólica $(\mathrm{mmHg})$ & $119 \pm 8$ \\
\hline Diastólica $(\mathrm{mmH} \mathrm{g})$ & $80 \pm 7$
\end{tabular}

\begin{tabular}{lc} 
Peso corporal $(\mathrm{kg})$ & $80.92 \pm 14.28$ \\
\hline Talla $(\mathrm{m})$ & $1.72 \pm 0.05$ \\
\hline $\mathrm{IMC}\left(\mathrm{kg} / \mathrm{m}^{2}\right)$ & $27.3 \pm 4.1$ \\
\hline Nivel de actividad física $(\mathrm{mMB})$ & $1.63 \pm 0.020$
\end{tabular}

frecuencia de obesidad tomando como punto de corte para la clasificación de sobrepeso un IMC de 27.8. ${ }^{35}$

En cuanto a la actividad física, los valores obtenidos mostraron que 29 sujetos se clasificaron como sedentarios; siete con actividad moderada, y únicamente dos con actividad intensa. La actividad física de los sujetos estuvo estrechamente vinculada con la ocupación que desempeñaban, desde trabajo de oficina (algunos realizaban ejercicio físico) hasta trabajo de albañilería.

\section{Estudio dietético}

Los datos de evaluación dietética (cuadro II) muestran que el consumo de fibra dietética en promedio fue de $37 \mathrm{~g} / \mathrm{d}$. Esta cantidad es levemente superior a la ingestión de seguridad establecida por el Instituto Nacional de la Nutrición Salvador Zubirán (INNSZ), ${ }^{38}$ que es de $35 \mathrm{~g} / \mathrm{d}$. Los principales alimentos aportadores fueron el frijol, la tortilla de maíz y la tortilla de harina de trigo. Estos alimentos coinciden con los principales aportadores de la dieta sonorense. ${ }^{39}$

Por otro lado, del total de la energía consumida, $35 \%$ proviene de grasa. Considerando que la Sociedad Mexicana de Nutrición y Endocrinología ${ }^{40}$ así como el National Cholesterol Education Program (NCEP) ${ }^{41}$ de los EUA, recomiendan que el porcentaje de grasa en la dieta no rebase el $30 \%$, la dieta aquí estudiada puede clasificarse como alta en grasa. De manera adicional, un análisis de la distribución de ácidos grasos mostró que $12 \%$ del total de grasa consumida proviene de ácidos grasos saturados; $14 \%$, de monoinsaturados, y $8 \%$, de polinsaturados.

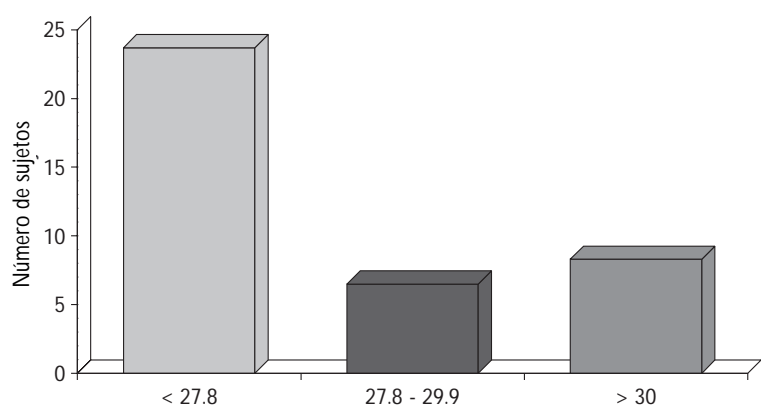

Figura 1. Distribución de obesidad en adultos nORmotensos. Hermosillo, Sonora, México, 1995-1996

\begin{tabular}{|c|c|c|c|}
\hline $\begin{array}{r}\text { Evaluación } \\
\text { Hermosil }\end{array}$ & $\begin{array}{r}\text { Cuadro } \\
\text { TÉTICA DE A } \\
\text { SONORA, N }\end{array}$ & $\begin{array}{l}\text { II } \\
\text { OLLTOS NORM } \\
\text { ÉXICO, } 1995\end{array}$ & $\begin{array}{l}\text { 1OTENSOS. } \\
\text {-1996 }\end{array}$ \\
\hline & Media $\pm \mathrm{DE}$ & $\begin{array}{c}\text { Recomendación } \\
\text { NAS* }\end{array}$ & $\begin{array}{c}\text { Recomendación } \\
\text { OMS }\end{array}$ \\
\hline Energía & & & \\
\hline (Kcal) & $2594 \pm 403$ & 2900 & 2900 \\
\hline (Kjoules) & $10743 \pm 1630$ & 12010 & 12010 \\
\hline Distribución de ene & & & \\
\hline Proteínas & $14.98 \pm 1.82$ & $15^{\S}$ & $10-15$ \\
\hline Grasa & $35 \pm 4.84$ & $<30^{\S}$ & $15-30$ \\
\hline Carbohidratos & $46 \pm 6.12$ & $45-50^{\S}$ & $55-75$ \\
\hline Alcohol & $3.88 \pm 3.87$ & - & - \\
\hline Sodio (mg) & $3753 \pm 1048$ & $<2400$ & $<4000$ \\
\hline Potasio (mg) & $2926 \pm 542$ & $2000-3500$ & - \\
\hline Calcio (mg) & $783 \pm 184$ & 800 & $400-500$ \\
\hline Fibra dietética (g) & $36.99 \pm 8.93$ & $35^{\#}$ & $16-24$ \\
\hline Colesterol (mg/d) & $443 \pm 218$ & $<300^{5}$ & $<300$ \\
\hline $\begin{array}{l}\text { * Referencia } 10 \\
\text { * Referencia } 9 \\
\text { § Referencia } 41 \\
\text { \# Referencia } 38\end{array}$ & & & \\
\hline
\end{tabular}

En lo referente a los micronutrimentos se encontró que el consumo de sodio ( $3753 \mathrm{mg}=9.4 \mathrm{~g}$ de sal) fue superior al recomendado. ${ }^{9,10} \mathrm{El}$ potasio estuvo dentro de los límites de seguridad y adecuación, ${ }^{10}$ en tanto que el calcio no logró cubrir la recomendación de 800 mg dada por NAS. ${ }^{10}$ 


\section{Presión arterial}

El análisis de regresión lineal simple efectuado (cuadro III) mostró que, de todas las variables dietéticas estudiadas, el consumo de sodio fue el único que presentó una relación lineal positiva altamente significativa con la presión diastólica.

En cuanto a la edad y la actividad física se refiere, éstas no mostraron ninguna asociación con la presión arterial.

El IMC fue la variable antropométrica que presentó una mejor relación con la presión diastólica. Asimismo, se encontró que de las variables evaluadas (independientes), las únicas que presentaron una relación significativa con la presión sistólica (cuadro III) fueron el peso y el IMC.

Dado que entre el peso y el IMC existe una fuerte correlación $(r=0.91 ; p=0.00)$ se consideró que no era necesario incluirlas en un modelo de regresión múltiple; para encontrar la variable que mejor explicara la variación de la presión sistólica se tomó al IMC con $r=$ 0.36 y $p=0.025$.

Para el caso de la presión diastólica, el IMC, la ingestión de sodio y la relación $\mathrm{Na}-\mathrm{K}$ fueron las varia- bles que se incluyeron en un modelo de regresión múltiple por pasos, y se encontró que la ingestión de sodio fue la que mejor explicó la variación de la presión diastólica (cuadro IV).

\section{Discusión}

Los sujetos estudiados presentaron un consumo de fibra dietética superior a lo que el INNSZ ${ }^{38}$ indica conveniente; ese valor es similar al notificado para una dieta rural mexicana ( $39.9 \mathrm{~g} /$ día) y muy superior al de una dieta urbana $\left(9.2 \mathrm{~g} /\right.$ día); ${ }^{42}$ también es mayor a las sugeridas por la $\mathrm{OMS}^{9}$ de $16-24 \mathrm{~g} / \mathrm{d}$ y a los informes de cifras recomendadas como adecuadas para poblaciones de los EUA y Canadá, en los que se propone un consumo de 25 y $30 \mathrm{~g} / \mathrm{d}_{1}{ }^{10}$ respectivamente. Al considerar esa información, puede decirse que la dieta de los sujetos estudiados es alta en fibra.

En este estudio no se encontró ningún efecto del consumo de fibra dietética en la presión diastólica y sistólica, lo cual coincide con lo notificado por otros autores $^{43}$ en sus estudios con sujetos que consumían dietas altas y bajas en fibra. Estas observaciones pudieran agregarse a la sugerencia de Anderson ${ }^{44}$ en el sen-

uadro III

MATRIZ de CORRELACIÓN DE PRESIÓN SISTÓliCA Y DIASTÓliCA DE ADULTOS NORMOTENSOS. Hermosillo, Sonora, México, 1995-1996

\begin{tabular}{|c|c|c|c|c|c|c|c|c|c|c|c|c|c|}
\hline $\begin{array}{l}\text { Presión } \\
\text { arterial }\end{array}$ & $\begin{array}{c}\text { Coeficiente de correlación } \\
\text { y nivel de significancia }\end{array}$ & Sodio & Potasio & Calcio & $\mathrm{Na}-\mathrm{K}$ & $\begin{array}{c}\text { Fibra } \\
\text { dietética }\end{array}$ & Energía & Alcohol & Colesterol & Edad & Peso & IMC & $\begin{array}{l}\text { Actividad } \\
\text { física }\end{array}$ \\
\hline \multirow[t]{2}{*}{ Sistólica } & $r$ & 0.276 & 0.146 & -0.038 & 0.2291 & 0.386 & 0.217 & 0.141 & 0.241 & -0.2949 & 0.357 & 0.362 & -0.162 \\
\hline & $p$ & 0.093 & 0.381 & 0.817 & 0.166 & 0.817 & 0.190 & 0.398 & 0.144 & 0.1384 & 0.027 & 0.025 & 0.330 \\
\hline \multirow[t]{2}{*}{ Diastólica } & $r$ & 0.430 & 0.258 & 0.089 & 0.293 & 0.036 & 0.270 & 0.127 & 0.060 & -0.095 & 0.304 & 0.339 & -0.038 \\
\hline & $p$ & 0.007 & 0.116 & 0.594 & 0.074 & 0.826 & 0.100 & 0.444 & 0.720 & 0.5667 & 0.062 & 0.037 & 0.816 \\
\hline
\end{tabular}

Cuadro IV

MOdelo PARA PRESIÓN DIASTÓLICA. EsTUdIO SOBRE COMPONENTES DIETÉTICOS Y PRESIÓN ARTERIAL. Hermosillo, Sonora, México, 1995-1996

\begin{tabular}{|c|c|c|c|c|c|c|c|c|c|c|c|}
\hline \multirow[b]{2}{*}{ Variables } & \multicolumn{5}{|c|}{ Modelo total } & & \multicolumn{5}{|c|}{ Modelo final } \\
\hline & Coeficiente & Error estándar & t-valor & $p$ & $\mathrm{R}^{2}$ & Variables & Coeficiente & Error estándar & t-valor & $p$ & $\mathrm{R}^{2}$ \\
\hline Constante & 54.8432 & 7.7540 & 7.0728 & 0.0000 & 0.2316 & Constante & 70.1017 & 3.5247 & 19.8883 & 0.0000 & 0.1849 \\
\hline IMC & 0.7085 & 0.2976 & 2.3804 & 0.0230 & & Ingestión de sodio & 0.0025 & 0.0008 & 2.8584 & 0.0070 & \\
\hline Ingestión de sodio & 0.003672 & 0.001309 & 2.8042 & 0.0083 & & & & & & & \\
\hline Relación $\mathrm{N}$ a/K & -5.9556 & 4.3251 & -1.3770 & 0.1775 & & & & & & & \\
\hline
\end{tabular}


tido de que la fibra dietética puede no ser el principal componente de una dieta vegetariana que contribuya al decremento de la presión sanguínea.

Por otro lado, la dieta evaluada también resultó ser alta en grasa; esta información coincide con la que indican otros estudios sobre población sonorense. ${ }^{39} \mathrm{La}$ dieta alta en grasa, aunada al elevado porcentaje de sujetos sedentarios, pudiera explicar en parte el sobrepeso y la obesidad que se observaron en $36.9 \%$ de los sujetos evaluados. Al respecto, no existen datos actualizados sobre obesidad que sean representativos de esa población y con los cuales se pueda hacer una comparación. Sin embargo, existe información de 1981 notificada por Valencia y colaboradores ${ }^{45}$ y que indica que se evaluó a un grupo de 140 sujetos de sexo masculino, 38 de los cuales se encontraban en el rango de 30-45 años, pertenecientes al nivel socioeconómico de bajo ingreso; $18.6 \%$ estaban por arriba de 27.7 de IMC en comparación con $36.9 \%$ de este estudio, utilizando el mismo punto de corte. Si bien los tamaños de muestra son muy pequeños, los datos parecen indicar una tendencia positiva al aumento del sobrepeso y la obesidad en estas comunidades.

Por otro lado, en este trabajo el IMC fue una de las variables que presentó una asociación altamente significativa con la presión diastólica, además de haber sido la que más afectó a la presión sistólica, por lo que el sobrepeso y la obesidad pudieran considerarse como uno de los principales factores de riesgo de que sujetos normotensos y aparentemente sanos padezcan hipertensión.

En relación con la actividad física no se encontró asociación significativa con la presión arterial. No obstante, se sabe que esta variable se relaciona directamente con el peso corporal ${ }^{46}$ (la relación encontrada en este estudio fue: $r=-0.3856$ y $p=0.01$ ), por lo que en este caso indirectamente puede estar jugando un papel importante debido a la asociación encontrada entre el grado de obesidad y la presión arterial.

El consumo de sodio se encontró $56 \%$ por arriba de la recomendación ${ }^{10}$ en $87 \%$ de los sujetos evaluados. La cantidad reportada se refiere únicamente a la propia de los alimentos sin considerar la aportada por la sal discrecional. Los resultados de este estudio difieren de lo indicado por el INNSZ, ${ }^{47}$ en el sentido de que mediante la excreción urinaria se estimó el consumo de sodio, siendo de $2.39 \mathrm{~g} / \mathrm{d}$ para una población rural y de $2.88 \mathrm{~g} / \mathrm{d}$ para una urbana.

El consumo de sodio fue la variable dietética que más efecto mostró sobre la presión diastólica. A pesar de que se consideró a los sujetos bajo estudio como normotensos, el alto consumo de sodio y el grado de obesidad podrían ser los principales factores de riesgo de padecer hipertensión.

Si bien la ingestión de potasio estuvo dentro de los límites de seguridad y adecuación, su consumo no alcanza los límites recomendados $(3500 \mathrm{mg} / \mathrm{d}$ en adelante) para prevenir la hipertensión. Lo anterior hace referencia a estudios llevados a cabo recientemente ${ }^{48} \mathrm{y}$ que demuestran con evidencia que una dieta alta en potasio reduce el riesgo de presión arterial causada por una dieta elevada en sodio, mientras que una ingestión baja-normal de potasio fomenta la aparición de alta presión inducida por el alto consumo de sodio.

En este contexto pudiera considerarse que la ingestión de potasio encontrada en este estudio fue bajanormal y que, aunada al alto consumo de sodio, podría favorecer la hipertensión. Sin embargo, no se encontró ningún efecto del consumo de este nutrimento sobre la presión arterial.

En cuanto al consumo de calcio, los sujetos evaluados ni siquiera lograron cubrir la recomendación. Lo anterior pudiera ser de riesgo como lo indican algunos estudios ${ }^{49}$ en los que se ha encontrado que existe una relación inversa entre el consumo de calcio y la presión arterial, así como también que un bajo consumo de este elemento puede ser predictor del estado de hipertensión. En este caso el calcio no mostró tener una relación con la presión arterial.

Otros componentes de la dieta que tampoco presentaron asociación alguna con la presión arterial fueron el colesterol y el alcohol.

\section{Conclusiones}

De los resultados anteriores se concluye que la elevada ingestión de sodio, así como el sobrepeso y la obesidad, presentan una asociación con la hipertensión arterial en sujetos normotensos. Dado que este estudio fue de corte transversal, no se puede concluir que dichas variables representen un factor de riesgo en esta condición clínica.

\section{Referencias}

1. Informe de la Comisión N acional Mixta Sobre la Detección, Evaluación y Tratamiento de la Hipertensión Arterial 1988. A teroma 1990;6:44-54.

2. $N$ ational Dairy Council. D ietary factors and blood pressure. Dairy Council Digest 1981;52:25-30.

3. Horan MJ, Lenfant C. Epidemiology of blood pressure and predictors of hypertension. Hypertension 1990;15 suppl i:20-24.

4. W einberger MH, Miller Jz, Luft FC, Grim CE, Fineberg N S. D efinitions and characteristics of sodium sensitivity and blood pressure resistance. Hypertension 1986;8 suppl ii:ii-127-ii134. 
5. Luft $\mathrm{F}$, W einberger $\mathrm{HH}$. Heterogeneus responses to changes in dietary salt intake:The salt-sensitivity paradigm. Am J Clin N utr 1997;65 suppl: S612-S617.

6. Cowley AW. Genetic and nongenetic determinants of salt sensitivity and blood pressure.Am J C lin N utr 1997;65 suppl:S587-S593.

7. Intersalt Cooperative Research Group. Intersalt:An international study of electrolyte excretion and blood pressure. Results for 24 hour urinary sodium and potassium excretion. Br Med J 1988;297:319-328.

8. Stampler J.The intersalt study: Background, methods, findings, and implications. Am J C lin N utr 1997;65suppl:S626-S642.

9.W orld Health 0 rganization. D iet, nutrition and the prevention of chronic diseases. Report of WHO Study Group. Technical Report Series 797. Geneva: W HO, 1990.

10. $\mathrm{N}$ ational A cademy of Science (NAS). Recommended dietary allowances. 10a. ed. W ashington, D.C .: N ational Academy Press, 1989.

11. Meneely GR, Batterbee HD. High potassium-low sodium environment and hypertension. Am J Cardiol 1976;38:768-785.

12. MacG regor GA. D ietary sodium and potassium intake and blood pressure. Lancet 1983;::750-753.

13. MacG regor GA, Smith SJ, Markander N D. Moderate potassium supplementation in essential hypertension. Lancet 1982;ii:567-570.

14. Khaw KT,Thom S. Randomised double blind cross-over trial of potassium on blood pressure in normal subjects. Lancet 1982;ii:1127-1129.

15. Krishna GG. Effect of potassium intake on blood pressure. J Am Soc Nephrol 1991;1:43-52.

16. MC Carron DA, Morris CD. Blood pressure response to oral calcium in persons with mild to moderate hypertension. Ann Intern Med 1985; 103:825-831

17. Karanja N , Mc Carron DA. Calcium and hypertension. Ann Rev N utr 1986;6:475-494.

18. Kromhout D, Bosschieter EB, Coulander CL. Potassium, calcium, alcohol intake and blood pressure:The zutphen study. Am J C lin N utr 1985; 41:1299-1304.

19. MC Carron DA, Morris C, Henry HJ, Stanton JL. Blood pressure and nutrient intake in the U nited States. Science 1984;224:1392-1398.

20. Anderson JW. Plant fiber and blood pressure. Ann Intern Med 1983; 842-846.

21. Weight A, Burstyn PG, Gibney MJ. Dietary fiber and blood pressure. Br Med J 1979;2:1541-1543.

22. Brussard JH, Van Raaij JM, Satasse-W olthius M, Katan MB, Haultrast $J G$. Blood pressure and diet in normotensive volunteers: Absence of effect of dietary fiber, protein, or fat. Am J C lin N utr 1981;34:2023-2029. 23. Luft FC, Miller JZ, Lyle RM, Melby CL, Fineberg N S, MC Carron DA. The effect of dietary interventions to reduce blood pressure in normal humans. J Am C lin N utr 1989;6:495-503.

24. Secretaría de Salud. Encuesta N acional de Enfermedades Crónicas. 2a. ed. México, D.F.: Secretaría de Salud, 1995.

25. Anuario estadístico. Diagnóstico de salud. Hermosillo: Servicios Médicos de Sonora, Dirección de Servicios de Salud, 1990.

26. C amberos M, G enesta MA, Huesca L. La pobreza en Sonora: Ios límites a la modernización. Rev Estudios Soc 1994;5(9):167-197.

27. Kuller LH. Invited commentary on "An epidemiology study of blood pressure levels in a bi-racial community in the Southern U nited States". Am J Epidemiol 1995;6:583-627.

28. Comstock GW. An epidemiologic study of blood pressure levels in a bi-racial community in the Southern United States. Am J Hyg 1957;65: 271-315.

29. Frolich ED, G rimm C, Labarthe DR, Maxwell MH. Recommendations for human blood pressure determination by sphygmomanometer. Hypertension 1988;11:210-222.
30. Bingham SA.The dietary assessment of individuals; methods, accuracy, new techniques and recommendations. Dietary Survey Methodology 1987;10:705-741.

31. Juvera F,Valencia ME, 0 rtega MI.Tablas de composición de alimentos en el noroeste de México: I Base de datos y II Programa Ciad AC. Memorias del X II Congreso de N utrición de Centroamérica y Panamá; 1990 jun 11-15; G uatemala, G uatemala.

32. Grijalva MI, Caire G, Sánchez A,Valencia ME. Composición química, fibra dietética y contenido de minerales en alimentos de consumo frecuente en el noroeste de México. A rch Latinoam N utr 1995; 45(2):145-150.

33. Hands E. Food Processor II Program. Salem (O R): Esha Research Editor, 1989.

34. Jelliffe DB, Jelliffe EPF. Community nutritional assessment; with special references to countries less technically developed. $N$ ueva York: 0 xford University Press, 1989.

35. Kuczmarski RJ. Prevalence of overweight and weight gain in the United States. Am J Clin N utr 1992;55:S495-S502.

36.Valencia ME, Mc N eill G, G onzález N L, Moya SY, Pinelli A, H aggarty BP. Energy expenditure during heavy work and its interaction with body weight. Bri J N utr 1997; 77:359-373.

37. FAO /O MS/UN U. N utrition Meeting Report. Series num. 724. Energy and protein requirement; Report of joint FAO/O MS/UNU expert consultation. Ginebra: O MS, 1985.

38. Bourges RH. La fibra al desnudo. Cuadernos N utr 1989;12(5):33-37. 39. Valencia ME, Hoyos LC, Ballesteros MN , O rtega MI, A tondo JL, Palacios D. Canasta estatal de consumo del estado de Sonora. Rev Estudios Soc. En prensa.

40. Sociedad Mexicana de Nutrición y Endocrinología, A.C. Diagnóstico y tratamiento de hiperlipidemias en México. Rev Mex Cardiol 1991;2(3): 97-105.

41. N ational Cholesterol Education Program. Expert panel on detection, evaluation, and treatment of high blood cholesterol in adults. JAMA 1993;269(23):3015-3023.

42. Rosado JL, López P, Morales M, Muñoz E, Allen LH. Bioavailability of energy, nitrogen, fat, zinc, iron and calcium from rural and urban Mexican diets. Br J N utr 1992;68:45-58.

43. Margetts $B M$, Beilin $L J$, Vandongen $R$, Armstrong BK. A randomized controlled trial of the effect of dietary fibre on blood pressure. Clin Sci 1987; 72:343-350

44.Anderson JW. Dietary fiber and human health. Hort Sci 1990; 25(12): 1488-1494.

45. Valencia ME, Saucedo S, Grijalva MI, Cruz R. Evaluación del estado nutricio en mujeres y hombres adultos en una zona urbana marginada de Sonora. Arch Latinoam N utr 1992;42(4):200.

46.Visser M, Laumer LJ, D eurenberg, D eeg DJH. Total and sports activity in older men and women: Relation with body fat. Am J Epidemiol 1997;145(8):752-761.

47. Sánchez CC, Solano ML, Flores J, Franklin MF, Limón N, Martínez V. Salt intake and blood pressure in rural and metropolitan Mexico. Arch Med Res 1996;27(4):559-566.

48. Tobian L. Dietary chloride and potassium have effects on the pathophysiology of hypertension in humans and animals. Am J C lin N utr 1997; 65 suppl:S606-S611.

49. Belizan JM,Villar J, Pineda O. Reduction of blood pressure with calcium suplementation in young adults. JAMA 1983;249:1161-1165. 